\title{
The role of UvrD in RecET-mediated illegitimate recombination in Escherichia coli
}

\author{
Kouya Shiraishi ${ }^{1,2}$, Yukiho Imai ${ }^{1,2}$, Shinji Yoshizaki ${ }^{1}$, Toshimasa Tadaki $^{1}$, \\ Yasuyuki Ogata ${ }^{3}$ and Hideo Ikeda ${ }^{1,2 *}$ \\ ${ }^{1}$ Institute of Medical Science, Medinet Inc., Tamagawadai 2-2-8, Setagaya-ku, \\ Tokyo 158-0096, Japan \\ ${ }^{2}$ Fundamental Research Laboratory, G\&G Science, Yokohama Leading \\ Venture Plaza 503, Ono-cho 75-1, Tsurumi-ku, \\ Yokohama 230-0046, Japan \\ ${ }^{3}$ Radioisotope Center, National Institute of Genetics, Yata 1111, \\ Mishima, Shizuoka 411-8540, Japan
}

(Received 23 May 2006, accepted 18 July 2006)

To study the mechanism of RecET-mediated illegitimate recombination, we examined the formation of $\lambda$ bio-transducing phage in Escherichia coli in the presence or absence of UV irradiation. We have previously reported that coexpression of RecE and RecT enhances the frequency of recA-independent illegitimate recombination. RecJOR proteins are required for this RecET-mediated illegitimate recombination, and $\operatorname{Rec} Q$ suppresses it. Here, we showed that the frequencies of both spontaneous and UV-induced RecET-mediated illegitimate recombination events are reduced by a $u v r D$ mutation. It should be noted that UvrD is required for illegitimate recombination only in the presence, but not in the absence, of RecET. In contrast, frequencies of RecET-mediated illegitimate recombination were not affected by $\operatorname{ruv} A B, \operatorname{ruv} C, \operatorname{rec} G$, and $\operatorname{rec} N$ mutations. The frequency of spontaneous and UV-induced illegitimate recombination in the uvrD recR double mutant was comparable to that of the $u v r D$ single mutant, suggesting that UvrD works at the same step as RecR in the RecET-mediated recombination pathway. Nucleotide sequence analyses of the recombination junctions showed that RecET-mediated illegitimate recombination detected in UvrD-deficient strain is short-homology-dependent. Based on these and previous results, we propose a model for the role of UvrD on RecET-mediated illegitimate recombination.

Key words: UvrD, RecET, illegitimate recombination, short-homology-dependent, recA-independent

In Escherichia coli, the main homologous recombination pathway requires the functions of the RecA and RecBCD proteins. The recombination and repair defects in $r e c B$ rec $C$ mutants can be suppressed by two kinds of mutations called $s b c A$ and $s b c B$ (Lloyd and Buckman, 1985; Templin et al., 1972). The $s b c A$ mutations induce expression of RecE and RecT proteins, which mediate homologous recombination through the RecE recombination pathway (Clark et al., 1993). This pathway also requires RecA, RecF, RecJ, RecO, RecR, RuvA, and UvrD (Gillen et al., 1981; Kuzminov, 1999; Luisi-DeLuca et al., 1989; Mendonca et al., 1993). The RecJ exonuclease and RecQ helicase are thought to process the double-stranded ends of DNA to produce 3' single-stranded DNA ends

Edited by Hideo Shinagawa

* Corresponding author. E-mail: ikeda@gandgscience.co.jp
(Clark and Sandler, 1994; Lovett and Kolodner, 1989). RecO promotes annealing of complementary singlestranded DNA ends and strand exchange in RecA-mediated homologous recombination (Luisi-DeLuca and Kolodner, 1994; Umezu and Kolodner, 1994). RecR protein, which is known to interact with $\mathrm{RecO}$ protein, enhances RecO protein's ability to stimulate displacement of SSB protein from ssDNA by RecA protein (Kantake et al., 2002). UvrD helicase is also thought to promote the generation of a 3' overhang by exonucleases (Matson et al., 1994).

Chromosomal aberrations are caused primarily by illegitimate recombination, which includes duplication, deletion, insertion, and translocation events. Illegitimate recombination occurs spontaneously at a low frequency, but it is greatly enhanced by treatment with UV light or other DNA damaging agents (Ikeda et al., 1995). These 
observations indicate that DNA lesions introduced by UV irradiation cause illegitimate recombination. There are two types of illegitimate recombination that lead to the formation of $\lambda$ bio-transducing phage: short-homology-dependent illegitimate recombination (SHDIR) and short-homology-independent illegitimate recombination (SHIIR). SHIIR occurs between sequences with virtually no homology and is mediated by DNA topoisomerases I or DNA gyrase (Shimizu et al., 1997; Ashizawa et al., 1999). SHDIR is induced by UV irradiation or other DNA-damaging agents and requires short regions of homology between recombination sites (Hanada et al., 2000; Shiraishi et al., 2005).

In SHDIR, the RecJ exonuclease promotes recombination, but RecQ helicase suppresses it (Ukita and Ikeda, 1996; Hanada et al., 1997). To explain roles of RecJQ in SHDIR, we have previously proposed a model of illegitimate recombination, the double-strand break-and-join model (Yamaguchi et al., 1995; Ukita and Ikeda, 1996; Hanada et al., 1997). In this model, RecJ may modify 5 protruding ends, which are produced by DNA doublestrand breaks (DSBs), to form blunt ends. RecQ DNA helicase may disrupt recombination intermediates formed by the annealing of DNA ends before the ligation step. This hypothesis is supported by the observation that RecQ helicase disrupts structures such as joint molecules or four-way junctions (Harmon and Kowalczykowski, 1998).

In recent studies, we found that coexpression of RecE and RecT enhances the frequency of SHDIR (Yamaguchi et al., 2000; Shiraishi et al., 2002). The recE gene encodes a 5' to 3' double-stranded-DNA-specific exonuclease, which selectively degrades the 5' end of DNA, producing a duplex with a 3' overhang (Joseph and Kolodner, 1983a; Joseph and Kolodner, 1983b). A model has been proposed in which this exonuclease produces 3' singlestrand at DNA ends, making them suitable for DNA-endjoining (Keim and Lark, 1990). RecT promotes annealing of complementary single DNA strands and can mediate ligation of the molecules (Hall et al., 1993; Hall and Kolodner, 1994). This model is consistent with our finding that RecET is involved in SHDIR. Furthermore, overexpression of ExoI suppresses this RecET-mediated illegitimate recombination (Yamaguchi et al., 2000). In addition, we found that $\mathrm{RecJ}, \mathrm{RecO}$, and RecR are required for the RecET-mediated illegitimate recombination, but RecA function is not (Shiraishi et al., 2002). We also found that RecQ plays a role in suppression of this recombination (Shiraishi et al., 2002).

The frequencies of illegitimate recombination during thermal induction of $E$. coli $\lambda$ cI857 ind $^{-}$lysogens were measured using the $\lambda \mathrm{Spi}^{-}$phage assay (Ikeda et al., 1995). Specialized transducing phage generated from the $\lambda$ prophage by illegitimate recombination usually contains the $E$. coli gene gal or bio, which is adjacent to the phage genome. Most of the transducing phages are defective in the red-gam region of their genome and can form plaques on an E. coli $\mathrm{P} 2$ lysogen lawn ( $\mathrm{Spi}^{-}$phenotype), whereas normal $\lambda$ phages cannot. Thus, it is possible to select $\lambda \mathrm{Spi}^{-}$phages from the phage pool. The number of $\lambda \mathrm{Spi}^{-}$phages is assumed to be the same as that of $\lambda$ bio transducing phages, since previous experiments have shown that most $\lambda \mathrm{Spi}^{-}$phages are $\lambda$ bio phages (Ikeda et al., 1995; Yamaguchi et al., 1995).

Since an E. coli K-12 strain, which was used as the wild-type, does not contain the $\operatorname{rec} E$ and $\operatorname{rec} T$ genes, we introduced a plasmid pRAC3, which carries the recE and rec $T$ genes, into the wild-type and mutant strains (Table 1). Unless otherwise indicated, we refer to the pRAC3transformed strains simply as the wild-type and mutant strains. The C-terminal domain of the recE gene and the full length of the rec $T$ gene are contained in pRAC3 (Luisi-DeLuca et al., 1988), but it maintains the functions that promote homologous recombination and illegitimate recombination (Takahashi et al., 1993; Yamaguchi et al., 2000).

To examine roles of Rec or Uvr functions in RecETmediated illegitimate recombination, we measured the frequency of $\lambda \mathrm{Spi}^{-}$phage production in $\operatorname{rec} G, \operatorname{recN}$, $\operatorname{ruvAB}$, ruvC or $u v r D$ mutants carrying pRAC3. The frequency of both spontaneous and UV-induced $\lambda$ Spi $^{-}$phage production was reduced in the $u v r D$-deficient strains compared to the wild-type strain (Table 2). On the other hands, frequencies of both spontaneous and UV-induced $\lambda \mathrm{Spi}^{-}$phage production in the $\operatorname{rec} G, \operatorname{recN}, \operatorname{ruv} A B$ and ruvC mutants were comparable to that of the wild-type strain (data not shown). It should be noted that UvrD is required for RecET-mediated illegitimate recombination, but not for illegitimate recombination in the absence of RecET.

In addition, we introduced the $u v r D$ mutation into the $r e c R$ mutant, and we measured the frequency of illegitimate recombination in the $u v r D$ recR double mutant. Frequency of spontaneous as well as UV-induced illegitimate recombination in the $u v r D$ recR double mutant was comparable to that of the $u v r D$ single mutant (data not shown). These results suggested that UvrD works at the same step as RecR in the RecET-mediated recombination pathway.

Next, we determined the distribution of recombination junctions in $\lambda \mathrm{Spi}^{-}$phage isolated from the RecETexpressing $u v r D$-deficient strain under UV-irradiation. Since illegitimate recombination takes place between $E$. coli bio-uvrB genes and $\lambda$ git-gam regions as shown in Fig. $1 \mathrm{~A}$, the recombination junctions were amplified by PCR with several primer oligonucleotide sets, followed by agarose gel electrophoresis analysis. We have previously reported that, in the phages derived from the RecETexpressing wild-type strain (HI2899) under UV-irradiation, most of the recombination takes place at one of the 
Table 1. E. coli strains used in this study

\begin{tabular}{|c|c|c|}
\hline Strains & Description & Source or construction \\
\hline Ymel & supE supF & Our collection \\
\hline WL95 & supE supF metB trpR hsdR tonA (P2) & Our collection \\
\hline HI2898 & 594 derivative, $\sup ^{0}\left(\lambda \operatorname{cI} 857^{a}\right) / \mathrm{pBR} 322$ & HI1162 \\
\hline HI2899 & 594 derivative, $\sup ^{0}\left(\lambda \operatorname{cI857^{a}}\right) / \mathrm{pRAC} 3\left(\operatorname{rec}^{+} T^{+}\right)^{\mathrm{b}}$ & HI1162 \\
\hline HI3155 & 594 derivative, sup ${ }^{0}$ recG258::Tn5 $\left(\lambda\right.$ cI85 $\left.7^{a}\right) / \mathrm{pBR} 322$ & HI3154 \\
\hline HI3156 & 594 derivative, sup $^{0}$ rec $G 258:: \operatorname{Tn} 5\left(\lambda\right.$ cI857 $\left.{ }^{a}\right) / \mathrm{pRAC} 3\left(r e c E^{+} T^{+}\right)^{\mathrm{b}}$ & HI3154 \\
\hline HI3121 & 594 derivative, sup $^{0}$ recN1502::Tn5 $\left(\lambda\right.$ cI857 $\left.{ }^{a}\right) / \mathrm{pBR} 322$ & HI3120 \\
\hline HI3122 & 594 derivative, sup $^{0}$ recN1502::Tn5 $\left(\lambda\right.$ cI857 $\left.{ }^{a}\right) / \mathrm{pRAC} 3\left(\text { rec }^{+} T^{+}\right)^{\mathrm{b}}$ & HI3120 \\
\hline HI3087 & 594 derivative, $\sup ^{0} \Delta \operatorname{ruvAB200::kan}\left(\lambda\right.$ cI857 $\left.^{a}\right) / \mathrm{pBR322}$ & HI2770 \\
\hline HI3088 & 594 derivative, $\sup ^{0} \Delta \operatorname{ruvAB200::kan}\left(\lambda \operatorname{cI857^{a}}\right) / \mathrm{pRAC} 3\left(r e c E^{+} T^{+}\right)^{\mathrm{b}}$ & HI2770 \\
\hline HI3089 & 594 derivative, sup $^{0} \Delta \operatorname{ruv} C 200::$ kan $\left(\lambda\right.$ cI857 $\left.{ }^{a}\right) / \mathrm{pBR} 322$ & $\mathrm{HI} 2771$ \\
\hline HI3090 & 594 derivative, $\sup ^{0} \Delta r u v C 200:: k a n\left(\lambda c I 857^{a}\right) / \mathrm{pRAC} 3\left(r e c E^{+} T^{+}\right)^{\mathrm{b}}$ & HI2771 \\
\hline HI3208 & AB1157 derivative, $\left(\lambda c I 857^{a}\right) / \mathrm{pBR} 322$ & HI2051 \\
\hline HI3209 & AB1157 derivative, $\left(\lambda c I 857^{a}\right) / \mathrm{pRAC} 3\left(r e c E^{+} T^{+}\right)^{\mathrm{b}}$ & HI2051 \\
\hline HI3238 & AB1157 derivative, $\Delta u v r D::$ tet $\left(\lambda\right.$ cI857 $\left.{ }^{a}\right) / \mathrm{pBR} 322$ & HI1521 $\times \lambda c I 857$ \\
\hline HI3239 & AB1157 derivative, $\Delta u v r D:: t e t\left(\lambda c I 857^{a}\right) / \mathrm{pRAC} 3\left(r e c E^{+} T^{+}\right)^{\mathrm{b}}$ & $\mathrm{HI} 1521 \times \lambda c I 857$ \\
\hline HI3276 & AB1157 derivative, $\Delta$ uvrD::tet recR252::Tn/Ominikan $\left(\lambda c I 857^{a}\right) / \mathrm{pBR} 322$ & HI3261 \\
\hline HI3277 & AB1157 derivative, $\Delta$ uvrD::tet recR252::Tn/Ominikan $\left(\lambda\right.$ cI857 $\left.{ }^{a}\right) / \mathrm{pRAC} 3\left(\text { recE }^{+} T^{+}\right)^{\mathrm{b}}$ & HI3261 \\
\hline
\end{tabular}

${ }^{a}$ All $\lambda$ cI857 ind $^{-}$lysogens contain one unit of $\lambda$ prophage.

${ }^{b}$ Plasmid pRAC3 is a pBR322 plasmid containing E. coli rec $E^{+} T^{+}$genes (Takahashi et al., 1993)

Table 2. Effect of the uvrD mutation on spontaneous or UV-induced RecET-mediated illegitimate recombination

\begin{tabular}{|c|c|c|c|c|c|c|c|}
\hline $\begin{array}{l}\text { UV dose } \\
\left(\mathrm{J} / \mathrm{m}^{2}\right)\end{array}$ & Strain $^{\mathrm{a}}$ & $\begin{array}{l}\text { Relevant } \\
\text { mutation }\end{array}$ & Plasmid & $\begin{array}{l}\mathrm{Spi}^{-} \mathrm{ph} \\
\text { total } \lambda\end{array}$ & $\begin{array}{l}\left(10^{-8}\right) \text { per } \\
\text { age }(\mathrm{SEM})^{\mathrm{b}}\end{array}$ & $\begin{array}{l}\text { Frequency relative } \\
\text { to control }\end{array}$ & $\begin{array}{l}\text { Burst size } \\
\text { (SEM) }\end{array}$ \\
\hline 0 & HI3208 & wild type & pBR322 & 0.13 & $(0.035)$ & 1 & $120(37)$ \\
\hline 0 & HI3238 & $\Delta$ uvrD::tet & pBR322 & 0.14 & $(0.025)$ & 1.1 & $72(19)$ \\
\hline 0 & HI3209 & wild type & pRAC3 $\left(r e c E^{+} T^{+}\right)$ & 2.2 & $(0.95)$ & 17 & $110(25)$ \\
\hline 0 & HI3239 & $\Delta$ uvrD::tet & pRAC3 $\left(r e c E^{+} T^{+}\right)$ & 0.17 & $(0.007)$ & 1.3 & $65(15)$ \\
\hline 10 & HI3208 & wild type & pBR322 & 2.9 & $(0.88)$ & 1 & $115(23)$ \\
\hline 10 & HI3238 & $\Delta u v r D:: t e t$ & pBR322 & 2.2 & (1.2) & 0.76 & $62(20)$ \\
\hline 10 & HI3209 & wild type & pRAC3 $\left(r e c E^{+} T^{+}\right)$ & 25 & (9) & 8.6 & 105 (19) \\
\hline 10 & HI3239 & $\Delta$ uvrD::tet & pRAC3 $\left(\right.$ rec $\left.E^{+} T^{+}\right)$ & 1.4 & $(0.72)$ & 0.48 & $55(18)$ \\
\hline 20 & HI3208 & wild type & pBR322 & 4.0 & $(0.95)$ & 1 & $112(15)$ \\
\hline 20 & HI3238 & $\Delta u v r D:: t e t$ & pBR322 & 3.2 & (1.6) & 0.8 & $47(15)$ \\
\hline 20 & HI3209 & wild type & pRAC3 $\left(r e c E^{+} T^{+}\right)$ & 47 & (2) & 12 & $102(13)$ \\
\hline 20 & HI3239 & $\Delta u v r D:: t e t$ & pRAC3 $\left(r e c E^{+} T^{+}\right)$ & 3.2 & (1.7) & 0.8 & $49(18)$ \\
\hline
\end{tabular}

${ }^{a}$ All strains are $E$. coli K-12 AB1157 or its derivatives containing one unit of $\lambda$ cI857 ind $^{-}$prophage. E. coli $\lambda$ cI857 $^{\text {ind }}{ }^{-}$ lysogen or its derivatives was grown to $1 \times 10^{8}$ cells $/ \mathrm{mL}$ and irradiated with UV at several dose. The lysogen was induced by incubation at $42^{\circ} \mathrm{C}$ for $15 \mathrm{~min}$ with shaking and then incubated at $37^{\circ} \mathrm{C}$ for $2 \mathrm{hr}$. The titer of $\lambda$ Spi $^{-}$phage was measured on a lawn of $E$. coli WL95 (P2). The number of total $\lambda$ phage was measured on a lawn of $E$. coli Ymel. The frequency of illegitimate recombination was calculated by dividing the number of $\lambda \mathrm{Spi}^{-}$phage by the total number of $\lambda$ phage (Ikeda et al., 1995).

${ }^{\mathrm{b}}$ All numbers are averages of four determinations.

${ }^{\mathrm{c}}$ Burst size indicates the total number of phages per cell. 


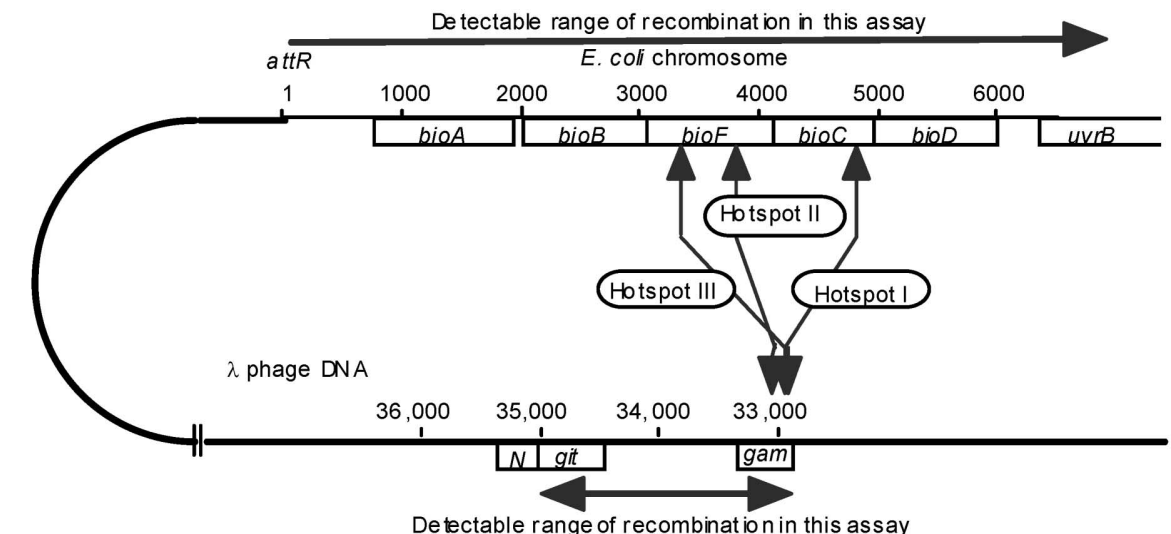

\section{B}

\section{(i) Hotspots}

\section{(a) Hotspot I}

UP V41, URA23

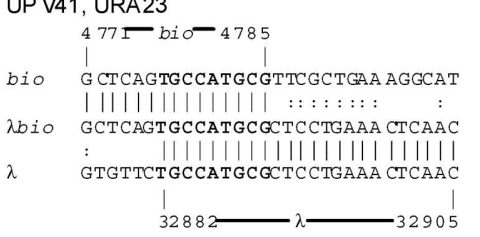

(b) Hotspot II

UPV19, URA12 $3725 \longrightarrow$ bio- 3746

bio aACCAGAATTGCTGGTAGTGACTTTT GGCA ||||||||||||||||||||||

$\lambda$ bio AACCAGAATTGC TGGTAGTGACGCGCCCAG : |||||||||||||||||||||| $\lambda \quad$ CGGGCGAGCTGC TGGTAGTGACGCGCCCAG (c) Hotspot III UP V33, UPV56

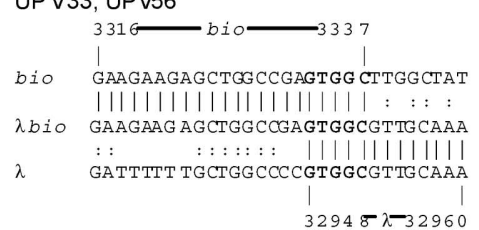

\section{(ii) Non-hotspots from uvrD- strains}

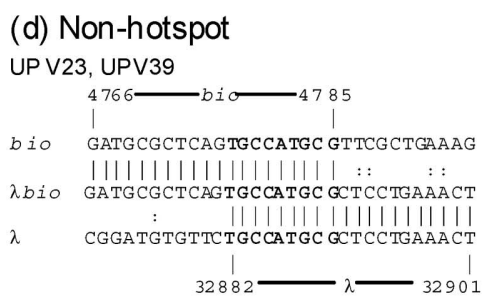

(g) Non-hotspot

URA48

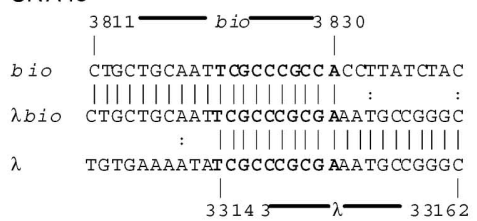

(e) Non-hotspot

UPV13

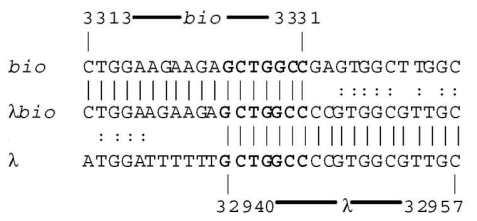

(f) Non-hotspot

URA52

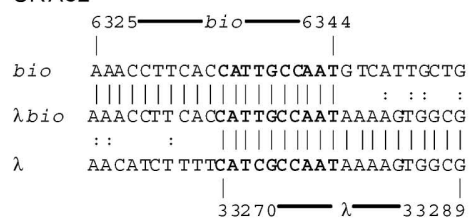

(h) Non-hotspot

URA51

$$
\begin{aligned}
& \begin{array}{l}
3693-\text { bio- } \\
\text { bio GGGGCGCGGCAGCTGCTGGCTGCAAA AGGT }
\end{array} \\
& \text { GGGGCGCGGCAGCTGCTGGCT } \\
& \text { nbio GGGGCGCGGCAGCTGCTGGTAGTGACGCGC }
\end{aligned}
$$

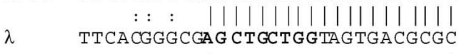

$$
\begin{aligned}
& { }_{33048-}-{ }_{\lambda 3067}
\end{aligned}
$$

Fig. 1. Nucleotide sequences of the recombination junctions of $\lambda$ bio transducing phages isolated from RecET-expressing and RecETnon-expressing strains under UV irradiation. The distributions of recombination sites of recombinant phages derived from various strains were determined by PCR. The junctions were classified into four classes: hotspot I, II, III, and non-hotspot. These junctions were sequenced with an ABI DNA sequencer. (A) Schematic representation of the recombination sites in this assay. (B, Panel a) Sequences of hotspot I detected at the recombination junctions of $\lambda$ bio transducing phages URA23, which were isolated from the RecET-expressing HI3239 uvrD mutants. Phages UPV41 were isolated from the RecET-non-expressing HI3238 uvrD mutants. Homologous sequences are shown in bold. Map coordinates for phage and bacterial sequences are indicated. (Panel b) Sequences of hotspot II detected at the recombination junctions of $\lambda$ bio transducing phages URA12, which were isolated from the RecET-expressing uvrD mutants. Phages UPV19 were isolated from the RecET-non-expressing uvrD mutants. (Panel c) Sequences of hotspot III detected in the recombination junctions of $\lambda$ bio transducing phages UPV33 and UPV56, which were isolated from the RecET-nonexpressing $u v r D$ mutant. (Panels $d$ and e) Sequences of non-hotspot sites detected in the recombination junctions of $\lambda$ bio transducing phages derived from the RecET-non-expressing uvrD mutant (HI3238). (Panels $f$ to $\mathrm{h}$ ) Sequences of non-hotspot sites detected in the recombination junctions of $\lambda$ bio transducing phages derived from the RecET-expressing uvrD mutant (HI3239).

three hotspots. The relative rates of recombination at hotspot I, II, and III were $46 \%, 4 \%$, and $34 \%$, respectively, indicating that RecET-mediated illegitimate recombina- tion is short-homology-dependent (Shiraishi et al., 2002). In this study, we tested the recombination junctions of 60 $\lambda \mathrm{Spi}^{-}$phages derived from the RecET-expressing $u v r D$ - 
deficient strain (HI3239) under UV-irradiation and found that the relative rates of recombination at hotspots I, II, and III were $72 \%, 10 \%$, and $0 \%$, respectively (Table3). The rate of recombination at hotspots III with the RecETexpressing uvrD-deficient strain (HI3239), was significantly low, compared with that with the RecET-expressing wild-type strain (HI2899). On the other hand, the rate of recombination at hotspot III with the RecET-nonexpressing $u v r D$-deficient strain (HI3238) was comparable with that with the RecET-non-expressing wild-type strain (HI2898) (Table 3). It is therefore suggested that UvrD is required for recombination at hotspot III with the RecET-expressing strain. Reason why recombination at hotspot III does not occur in the $u v r D$-deficient strain has not been known yet.

The junctions of the $\lambda$ bio transducing phages UPV41 and URA23 were produced by recombination at hotspot I, which was previously found by Yamaguchi et al. (Yamaguchi et al., 1995). At this hotspot, the recombination sites are known to share a 9-bp region of homology, as shown in Fig. 1B, panel a. The hotspot sites II and III share $13 \mathrm{bp}$ and $5 \mathrm{bp}$ regions of homology, respectively, as shown in Fig. 1B, panel $\mathrm{b}$ and c. Panels $\mathrm{d}$ and e in Fig. $1 \mathrm{~B}$ show the junction sequences of $\lambda$ bio transducing phages derived from recombination at non-hotspot sites in the RecET-non-expressing $u v r D$-deficient strain (average length of homology, $9.3 \mathrm{bp}$ ), and panels $\mathrm{f}$ to $\mathrm{h}$ show the junction sequences of $\lambda b i o$ transducing phages derived from recombination at non-hotspot sites in the RecET-expressing $u v r D$-deficient strain (average length of homology, $9.5 \mathrm{bp}$ ). All recombination sites shared short regions of homology between $E$. coli and $\lambda$ phage DNA. These results indicate that short regions of homology also have a role in illegitimate recombination in the absence of UvrD function.

Early studies of illegitimate recombination suggested that 3' single-stranded overhangs were required to promote the recombination (Allgood and Silhavy, 1991; Yamaguchi et al., 2000). Here, we have shown that UvrD helicase is required for RecET-mediated illegitimate recombination, but not for illegitimate recombination in the absence of RecET. The UvrD protein has a
$3 \rightarrow 5$ ' DNA helicase activity and is suggested to play a role in processing of DNA ends in combination with nucleases (Matson et al., 1994). This is consistent with a model in which UvrD helps the RecE exonuclease to produce 3' single-strand overhangs, thus increasing the frequency of illegitimate recombination.

In homologous recombination, $\mathrm{RecO}$, RecR, and possibly RecF proteins have been shown to promote formation of RecA nucleoprotein filaments on single-stranded DNA (ssDNA) gaps covered with single-stranded DNA binding protein (SSB), suggesting that RecFOR help RecA to overcome inhibition by SSB (Umezu et al., 1993). In addition, Veaute et al. (2005) suggested that UvrD helicase dismantles RecA nucleoprotein filaments, thus preventing homologous recombination. Namely, RecFOR and UvrD might have adverse roles in homologous recombination. These results raise another possibility that UvrD indirectly enhances the RecET-mediated illegitimate recombination by preventing RecAFOR-mediated homologous recombination. However our present studies showed that the frequency of illegitimate recombination was reduced in the recR-or $u v r D$-deficient strains with pRAC3, compared to those in the wild-type strain. Furthermore, the frequency of illegitimate recombination in the $u v r D$ recR double mutant with pRAC3 was comparable with those in the wild-type strains. These results suggested that UvrD works at the same step as RecR in the RecET-mediated illegitimate recombination. Therefore the above possibility seems to be unlikely.

We have previously reported that co-expression of RecE and RecT enhances the frequency of spontaneous and UVinduced illegitimate recombination (Shiraishi et al., 2002). Based on these and the previous results, we propose the following model for RecET-mediated illegitimate recombination. DNA double-strand breaks produce DNA ends which have long 5' single-stranded overhangs. RecJ digests these 5' single-strands, forming blunt ends. RecE digests the 5' single-strands of the blunt ends, producing 3 ' single-stranded overhangs, and UvrD may facilitate the generation of 3' single-stranded overhangs by the RecE exonuclease. These 3' overhangs might bind with SSB and then degraded by Exonuclease I, a single-

Table 3. Summary of distribution of recombination sites of $\lambda$ bio transducing phages formed following UV irradiation ${ }^{\mathrm{a}}$

\begin{tabular}{rllccrcc}
\hline \hline Strain & Relevant & Plasmid & Number of & \multicolumn{3}{c}{ Rate of recombination (Rate with 95\% probability) at: } \\
& mutation & & phages tested & HotI (\%) & HotII (\%) & HotIII (\%) & Non-hot (\%) \\
\hline HI2898 $^{\text {b }}$ & wild type & pBR322 & 48 & $33(19-52)$ & $21(10-39)$ & $2(0.2-10)$ & $44(27-66)$ \\
HI2899 $^{\text {b }}$ & wild type & pRAC3 & 50 & $46(29-69)$ & $4(0.5-14)$ & $34(20-54)$ & $16(7-31)$ \\
HI3238 & uvrD & pBR322 & 60 & $75(54-95)$ & $3(0.3-12)$ & $5(0.6-16)$ & $17(8-32)$ \\
HI3239 & uvrD & pRAC3 & 60 & $72(51-96)$ & $10(3.7-21)$ & $0(0.1-3.7)$ & $18(9-33)$ \\
\hline
\end{tabular}

a The distribution of recombination sites of recombinant phages derived from the uvrD strain was determined by PCR.

The junctions were classified into four classes: hotspot I (Hot I), II (Hot II), III (Hot III), and non-hotspot (Non-hot).

The dose of UV irradiation was $20 \mathrm{~J} / \mathrm{m}^{2}$.

b The data with HI2898 and HI2899 were cited from Shiraishi et al. (2002). 
stranded DNA-specific nuclease, whose activity is stimulated by SSB binding to ssDNA (Molineux and Gefter, 1975). A role of RecT might be to compete with SSB for binding to the 3 ' single-stranded overhangs, thus protecting them from degradation by ExoI. In addition, RecO and RecR are known to bind to SSB-coated ssDNA without displacing SSB (Umezu and Kolodner, 1994). A possible role of RecOR could also be the protection of the 3' single-stranded overhangs from digestion by ExoI. Since both RecT and $\mathrm{RecO}$ are known to have annealing activities of ssDNA, these proteins may promote annealing of complementary ssDNA, forming hydrogen-bonded intermediates. Finally DNA ligase would play a role in joining of the hydrogen-bonded intermediates.

We thank Dr. S. W. Matson for providing E. coli strains and Dr. K. Egawa for encouragement. This study was supported by Grants-in-Aid for Scientific Research on Priority Areas (B) to Y.O. from the Ministry of Education, Culture, Sports, Science, and Technology of Japan.

\section{REFERENCES}

Allgood, N. D., and Silhavy, T. (1991) Escherichia coli xonA $(s b c B)$ mutants enhance illegitimate recombination. Genetics 127, 671-680.

Ashizawa, Y., Yokochi, T., Ogata, Y., Shobuike, Y., Kato, J., and Ikeda, H. (1999) Mechanism of DNA gyrase-mediated illegitimate recombination: characterization of Escherichia coli gyrA mutations that confer hyper-recombination phenotype. J. Mol. Biol. 289, 447-458.

Clark, A. J., Sharma, V., Brenowitz, S., Chu, C. C., Sandler, S., Satin, L., Templin, A., Berger, I., and Cohen, A. (1993) Genetic and molecular analyses of the C-terminal region of the recE gene from the Rac prophage of Escherichia coli K12 reveal the recT gene. J. Bacteriol. 175, 7673-7682.

Clark, A. J., and Sandler, S. (1994) Homologous genetic recombination: the pieces begin to fall into place. Crit. Rev. Microbiol. 20, 125-142.

Gillen, J. R., Willis, D., and Clark, J. A. (1981) Genetic analysis of the RecE pathway of genetic recombination in Escherichia coli K-12. J. Bacteriol. 145, 521-532.

Hall, S. D., Kane, M., and Kolodner, D. R. (1993) Identification and characterization of the Escherichia coli RecT protein, a protein encoded by the recE region that promotes renaturation of homologous single-stranded DNA. J. Bacteriol. 175, 277-287.

Hall, S. D., and Kolodner, R. (1994) Homologous pairing and strand exchange promoted by the Escherichia coli RecT protein. Proc. Natl. Acad. Sci. USA 91, 3205-3209.

Hanada, K., Ukita, T., Kohno, Y., Saito, K., Kato, J., and Ikeda, H. (1997) RecQ DNA helicase is a suppressor of illegitimate recombination in Escherichia coli. Proc. Natl. Acad. Sci. USA 94, 3860-3865.

Hanada, K., Iwasaki, M., Ihashi S., and Ikeda, H. (2000) UvrA and UvrB suppress illegitimate recombination: synergistic action with RecQ helicase. Proc. Natl. Acad. Sci. USA 97, 5989-5994.

Harmon, F. G., and Kowalczykowski, S. C. (1998) RecQ helicase, in concert with RecA and SSB proteins, initiates and disrupts DNA recombination. Genes Dev. 12, 1134-1144.

Ikeda, H., Shimizu, H., Ukita, T., and Kumagai, M. (1995) A novel assay for illegitimate recombination in Escherichia coli: stimulation of lambda bio transducing phage formation by ultra-violet light and its independence from RecA function. Adv. Biophys. 31, 197-208.

Joseph, J. W., and Kolodner, R. (1983a) Exonuclease VIII of Escherichia coli. I. Purification and physical properties. J. Biol. Chem. 258, 10411-10417.

Joseph, J. W., and Kolodner, R. (1983b) Exonuclease VIII of Escherichia coli. II. Mechanism of action. J. Biol. Chem. 258, 10418-10424.

Kantake, N., Madiraju, V. M., Sugiyama, T., and Kowalczykowski, S. C. (2002) Escherichia coli RecO protein anneals ssDNA complexed with its cognate ssDNA-binding protein: A common step in genetic recombination. Proc. Natl. Acad. Sci. USA. 99, 15327-15332.

Keim, P. and Lark, K. G. (1990) The RecE recombination pathway mediates recombination between partially homologous DNA sequences: structural analysis of recombination products. J. Struct. Biol. 104, 97-106.

Kuzminov, A. (1999) Recombinational repair of DNA damage in Escherichia coli and bacteriophage lambda. Microbiol. Mol. Biol. Rev. 63, 751-813.

Lloyd, R. G., and Buckman, C. (1985) Identification and genetic analysis of $s b c C$ mutations in commonly used $r e c B C s b c B$ strains of Escherichia coli K-12. J. Bacteriol. 164, 836844.

Lovett, S. T., and Kolodner, R. (1989) Identification and purification of a single-stranded-DNA-specific exonuclease encoded by the recJ gene of Escherichia coli. Proc. Natl. Acad. Sci. USA 86, 2627-2631.

Luisi-DeLuca, C., Clark, J. A., and Kolodner, D. R. (1988) Analysis of the recE locus of Escherichia coli K-12 by use of polyclonal antibodies to exonuclease VIII. J. Bacteriol. 170, 5797-5805.

Luisi-DeLuca, C., Lovett, S., and Kolodner, D. R. (1989) Genetic and physical analysis of plasmid recombination in $\mathrm{recB}$ rec $C$ $s b c B$ and $r e c B$ recC $s b c A$ Escherichia coli K-12 mutants. Genetics 122, 269-278.

Luisi-DeLuca, C., and Kolodner, R. (1994) Purification and characterization of the Escherichia coli RecO protein. Renaturation of complementary single-stranded DNA molecules catalyzed by the RecO protein. J. Mol. Biol. 236, 124-138.

Matson, S. W., Bean, D., and George, W. J. (1994) DNA helicases: enzymes with essential roles in all aspects of DNA metabolism. Bioessays 16, 13-22.

Mendonca, V. M., Kaiser-Rogers, K., and Matson, S. W. (1993) Double helicase II (uvrD)-helicase IV (helD) deletion mutants are defective in the recombination pathways of Escherichia coli. J. Bacteriol. 175, 4641-4651.

Molineux, I. J. and Gefter, M. L. (1975) Properties of the Escherichia coli DNA-binding (unwinding) protein interaction with nucleolytic enzymes and DNA. J. Mol. Biol. 98, 811-825.

Onda, M., Hanada, K., Kawachi, H., and Ikeda, H. (1999) Escherichia coli MutM suppresses illegitimate recombination induced by oxidative stress. Genetics 151, 439-446.

Shimizu, H., Yamaguchi, H., Ashizawa, Y., Kohno, Y., Asami, M., Kato, J., and Ikeda, H. (1997) Short-homology-independent illegitimate recombination in Escherichia coli: distinct mechanism from short-homology-dependent illegitimate recombination. J. Mol. Biol. 266, 297-305.

Shiraishi, K., Hanada, K., Iwakura, Y., and Ikeda, H. (2002) Roles of RecJ, RecO, and RecR in RecET-mediated illegitimate recombination in Escherichia coli. J. Bacteriol. 184, 4715-4721.

Takahashi, N. K., Kusano, K., Yokochi, T., Kitamura, Y., 
Yoshikura, H., and Kobayashi, I. (1993) Genetic analysis of double-strand break repair in Escherichia coli. J. Bacteriol. 175, 5176-5185.

Templin, A., Kushner, S., and Clark, J. A. (1972) Genetic analysis of mutations indirectly suppressing $\mathrm{rec} B$ and $\mathrm{rec} C$ mutations. Genetics 72, 105-115.

Ukita, T., and Ikeda, H. (1996) Role of the recJ gene product in UV-induced illegitimate recombination at the hotspot. J. Bacteriol. 178, 2362-2367.

Umezu, K., Chi, W.N., and Kolodner, D.R. (1993) Biochemical interaction of the Escherichia coli RecF, RecO, and RecR proteins with RecA protein and single-stranded DNA binding protein. Proc. Natl. Acad. Sci. USA 90, 3875-3879.

Umezu, K., and Kolodner, R. D. (1994) Protein interactions in genetic recombination in Escherichia coli. Interactions involving $\mathrm{RecO}$ and RecR overcome the inhibition of RecA by single-stranded DNA-binding protein. J. Biol. Chem. 269, 30005-30103.

Veaute, X., Delmas, S., Selva, M., Jeusset, J., Le Cam, E., Matic, I., Fabre, F., and Petit, M. A. (2005) UvrD helicase, unlike Rep helicase, dismantles RecA nucleoprotein filaments in Escherichia coli. EMBO J. 24, 180-189.

Yamaguchi, H., Yamashita, T., Shimizu, H., and Ikeda, H. (1995) A hotspot of spontaneous and UV-induced illegitimate recombination during formation of lambda bio transducing phage. Mol. Gen. Genet. 248, 637-643.

Yamaguchi, H., Hanada, K., Asami, Y., Kato, J., and Ikeda, H. (2000) Control of genetic stability in Escherichia coli: the SbcB 3'-5' exonuclease suppresses illegitimate recombination promoted by the RecE 5'-3' exonuclease. Genes Cells 5, 101-109. 